\title{
ENSEÑANZA-APRENDIZAJE CON WEB 2.0 Y 3.0
}

María Belén Vaquerizo-García1. Universidad de Burgos. España belvagar@ubu.es

\section{RESUMEN}

La Web 2.0 como la Web 3.0 o Web Semántica constituyen un avance importante en el área educativa ofreciendo grandes posibilidades en la educación a distancia y en espacios virtuales educativos. En el contexto de la enseñanza-aprendizaje favorecen el aprendizaje autónomo y colaborativo de los alumnos y el desarrollo en ellos de nuevas capacidades y competencias. En el ámbito educativo, en los aspectos tecnológico y social, aportan un conjunto de estrategias y funcionalidades sofisticadas de publicación y de gestión de contenidos educativos en entornos Web. Además, junto con la aparición de herramientas con licencia de uso libre, sirve de ayuda a los docentes en el desarrollo de contenidos educativos interactivos y multimedia adecuados para la formación online de forma autónoma; estos contenidos se pueden organizar en sitios Web, para su utilización en un espacio virtual. El presente trabajo muestra una experiencia del uso de estas herramientas en la impartición de una asignatura.

PALABRAS CLAVE: Web 2.0 - Web 3.0 - Aprendizaje Autónomo - Aprendizaje Colaborativo - Herramientas Didácticas. 


\title{
TEACHING-LEARNING WITH WEB 2.0 AND 3.0
}

\begin{abstract}
Web 2.0 and the Web 3.0 or Semantic Web constitutes an important advance in the educational area offering high possibilities in distant education and in virtual educational spaces. In the context of teaching-learning, both of them help in the autonomous and collaborative learning of the students and in the development of theirs capacities. In the field of education, in the aspects technological and social, they give a set of strategies and sophisticated functionalities of publication and management of educational contents in Web environments. In addition, with the existence of tools with license of free use, they can help to the teachers in the development of educational interactive contents and multimedia suitable for the formation online in an autonomous way; these contents can be organized in web sites, for his use in a virtual space. The present work shows an experience of the use of these tools in the teaching of a subject.
\end{abstract}

KEY WORDS: Web 2.0 - Web 3.0 - Autonomous Learning - Collaborative Learning Didactic Tools.

\section{INTRODUCCIÓN}

La Web 2.0 y la Web 3.0, mediante el uso de herramientas orientadas hacia un nuevo modelo de aprendizaje y enseñanza, propician el desarrollo de nuevas capacidades y competencias en el alumnado (colaboración, equipos de trabajo, etc). Bajo este supuesto, en este trabajo se presenta una experiencia docente para el desarrollo de las prácticas de una asignatura en un espacio virtual, en cuanto al diseño de una estrategia que se adecue a los medios disponibles y asegure el éxito en el aprendizaje autónomo y colaborativo de los alumnos, al permitirles seguir su propio ritmo de aprendizaje.

Cabe indicar, que aunque en este trabajo solo se estén abordando las bondades que en el contexto docente ofrecen ambas Web, hay que tener presente que no es suficiente el simple uso de la Web, pues en ello, se ha de considerar la necesidad de modelos pedagógicos, didácticos y requerimientos tecnológicos. Así, es sumamente importante en el contexto educativo, el desarrollo adecuado del material didáctico, la configuración de una metodología de trabajo (estableciendo unas pautas de pedagogía y prototipos de participación de los alumnos, todo ello coordinado y dirigido por el profesor) y la elección adecuada de herramientas para la creación de contenidos. 


\section{METODOLOGÍA}

El estudio emplea el método analítico-sintético para analizar la Web 2.0, la Web 3.0 o Web Semántica como una herramienta de enseñanza a distancia en una asignatura.

\section{ANÁLISIS Y DISCUSIÓN}

\subsection{Web 2.0 y Web 3.0}

En la Web 2.0, referida como una Web colaborativa, el contenido lo crean los usuarios, lo que ha supuesto una revolución en la difusión de la información. Implica una mayor participación y relación entre los alumnos y el profesor, y esto se puede analizar desde dos aspectos, el tecnológico y el social. En el aspecto tecnológico, la Web 2.0 permite disponer de un conjunto de herramientas sofisticadas de publicación y gestión de contenidos. En el aspecto social, posibilita la aparición de una inteligencia colectiva a partir de la agregación de aportaciones individuales no sistematizadas ni guiadas explícitamente.

Algunas aplicaciones útiles en docencia de estas herramientas se muestran en la siguiente

Tabla 1. Algunas aplicaciones docentes de las Herramientas de la Web 2.0.

\begin{tabular}{|c|c|c|}
\hline \multicolumn{3}{|c|}{ Algunas aplicaciones de las Herramientas Web 2.0 a la Docencia } \\
\hline $\begin{array}{l}\text { - Blog } \\
\text { - Wiki } \\
\text { - Foros } \\
\text { - Calendario en línea } \\
\text { - Correo electrónico web } \\
\text { - Podcast (archivos de audio) } \\
\text { - Portafolio electrónico } \\
\text { - Edición imagen en línea } \\
\text { - Videoconferencia } \\
\text { - Cuestionarios en línea } \\
\text { - Autoevaluación en línea }\end{array}$ & $\begin{array}{l}\text { - Edición de vídeo en línea } \\
\text { - Edición de audio (audacity, garageband, etc.) } \\
\text { - Repositorio y compartir imagen (flickr, picasa, etc.) } \\
\text { - Red social (ning, facebook, etc.) } \\
\text { - Mundos virtuales (second life, lively, etc.) } \\
\text { - Ofimática en línea (procesar texto, hoja de cálculo, etc) } \\
\text { - Página personalizada }\end{array}$ & $\begin{array}{l}\text { - Marcadores sociales (del.icio.us) } \\
\text { - Búsqueda mediante etiquetas } \\
\text { - Gestión bibliográfica } \\
\text { - Librerias digitales colaborativas } \\
\text { - Lectores de feeds (agregadores de } \\
\text { información) } \\
\text { - Otras }\end{array}$ \\
\hline
\end{tabular}

De entre estas aplicaciones docentes en la experiencia docente que se trata en el presente trabajo se ha trabajado con Blogs y Wikis. Los blogs o bitácoras son páginas Web donde se permiten crear, editar y publicar de manera fácil "información" en orden cronológico, o bien por categorías y suelen contener además una lista de enlaces a otros sitios Web de interés del autor. En cuanto a una Wiki se refiere, se puede decir que es más un sistema de catalogación de contenidos y documentación que facilita la escritura colaborativa. 
Por otra parte, en la Web 3.0 o Web semántica se parte de la base de añadir información adicional para complementar un contenido e incrementar el significado de los datos, logrando una mayor interoperatividad. Esta Web ha surgido del cambio en la forma de las relaciones sociales y comunicación entre las personas, como es el caso de las redes sociales.

El propósito de la Web Semántica a nivel educativo, es lograr agentes software que interpreten el significado de los contenidos de la Web, para ayudar a los usuarios a desarrollar sus tareas.

\subsection{Experiencia Docente con Web 2.0 y 3.0}

La experiencia se realizó en la impartición de las prácticas de una asignatura de Ingeniería del Software de $2^{\circ}$ Curso de I.T.I.G (plan antiguo), con un grupo de aproximadamente 40 alumnos. Los alumnos se agruparon en grupos de 4 y a cada grupo se le asignó un problema concreto a resolver sobre el que realizar las fases de desarrollo del Software, bajo la metodología Métrica V.3. Se trata de promover la creación de grupos de alumnos activos, utilizando, para ello, espacios de trabajo y comunicación para la actividad grupal.

Se ha propuesto una didáctica a aplicar mediante el uso de Blogs y Wikis a nivel de cada grupo de alumnos y una comunidad de trabajo creada previamente por el profesor común para todos los alumnos. En la comunidad se ha de disponer de información y recursos comunes a todos los grupos de alumnos, y de sistemas de comunicación interna y externa, foro, chat, así como información sobre las herramientas posibles a utilizar para el desarrollo de los contenidos.

Además, siguiendo un calendario establecido por el profesor, han de ir haciendo entregas de cada una de las partes de las que consta el trabajo, por ello, existe una división por fases o bloques conceptuales comunes a todos los problemas. Mediante estas herramientas se establecen puntos de encuentro entre alumnos y profesor a nivel de cada parte de la práctica a resolver, y donde el tutor podrá ir respondiendo a las preguntas que los integrantes del grupo realicen sobre el material que se vaya compartiendo. De este modo, en el apartado del Blog correspondiente a un bloque conceptual, pueden existir dudas de varios grupos de alumnos diferentes, así como respuestas o soluciones que el profesor aporta y que pueden ser comunes a varios grupos de alumnos.

\subsection{Resultados de la Experiencia Docente}

Con este planteamiento didáctico la enseñanza no se ha centrado en impartir contenidos, sino en utilizar esos contenidos para ayudar al alumno en el proceso del desarrollo de sus competencias personales y profesionales. En base a los resultados 
con cursos anteriores, y, la otra vertiente es en base a unas encuestas de satisfacción realizadas a los alumnos, donde han dejado constancia de que este modelo de aprendizaje les ha facilitado la adquisición de los conocimientos.

Además, también se ha favorecido el proceso de evaluación, ya que se proporciona mucha información de la actividad realizada por los alumnos. La evaluación se ha realizado desde dos enfoques: evaluar los resultados finales de los alumnos, y evaluar el curso en-línea como actividad formativa (sobre la participación de los alumnos en cuanto a actitudes, contribuciones y resultados).

Por otra parte, cabe tener en cuenta que estas herramientas son intrínsecamente colaborativas, pero los sistemas actuales de evaluación son esencialmente individualistas. Normalmente, en la evaluación, suele ser complicado el poder determinar la contribución de un individuo al trabajo del grupo. Por ello, en todo momento el profesor debe tener un mecanismo continuo de seguimiento y revisión de los trabajos presentados, etc.

\section{CONCLUSIONES}

Desde el punto de vista del alumno, se espera que el uso de esta estrategia didáctica, por una parte ayude le ayude a comprender la importancia del conocimiento de la materia para su futuro profesional, y, por otra parte, contribuya a desarrollar en los alumnos diferentes capacidades, tales como: Capacidad de análisis y síntesis, Capacidad de organización y planificación, Aprendizaje autónomo.

Por otra parte, desde el punto de vista del profesor, le ha de servir como ayuda en la planificación temporal y en la valoración del esfuerzo por parte del alumno. Para ello, ha de estructurar los contenidos basándose en tareas reales que el alumno ha de resolver. Esto le exige un notable esfuerzo, pues se requiere que el Blog creado en la comunidad esté bien planificado, con propuestas bien elaboradas y con recursos significativos.

\section{REFERENCIAS}

Cano, R. (2009). Tutoría universitaria y aprendizaje por competencias. ¿Cómo lograrlo?. REIFOP, 12 (1): 181-204.

Correa, J. \& DE Pablos, J. (2009). Nuevas Tecnologías e Innovación Educativa. Revista de Psicodidáctica, 14 (1): 133-145.

Cuesta Morales, P. (2008). Utilizando herramientas de la Web 2.0 en la Adaptación de la Materia Sistemas Multiagente al EEES. Jenui. 
Ferro, C.; Martinez, A; Otero, M.C. (2009). Ventajas del uso de las TICs en el proceso de enseñanza-aprendizaje desde la óptica de los docentes universitarios españoles. Revista Electrónica de Tecnología Educativa, 29.

Freire, J. (2007). La Web 2.0 como plataforma para el aprendizaje activo y colaborativo.

Gewerc Barujel, A. (2005). El uso de weblogs en la docencia universitaria. Revista Latinoamericana de Tecnología Educativa.

Griol, D; Molina J. M; Callejas, Z.; López-Cózar, R. (2011). La plataforma EDUCAGENT: Agentes Conversacionales Inteligentes y Entornos Virtuales aplicados a la Docencia. Revista Iberoamericana de Informática Educativa.

Hernández, F. (2005).Enseñar y aprender en la Universidad: Una adaptación necesaria de las titulaciones al Espacio Europeo de Educación Superior. Revista Circunstancia, 8.

Mauri Majós, T; Colomina Alvarez, R; \& Gispert, I. (2009). Diseño de propuestas docentes con TIC para la enseñanza de la autorregulación en la Educación Superior. Revista de Educación, 348: 377-399.

Narváez, J. L., Périssé, M. C. (2011). La Web Semántica en la Educación Superior. Revista académica Hologramática.

Ramírez León, Y., Peña Arcila, J. B.. (2011). Web 3.0 as a tool to Support Distance Education. Granada.

Salinas, J.; Aguaded, J. I. \& Cabero, J. (2004). Tecnologías para la educación. Diseño, producción y evaluación de medios para la formación docente. Madrid: Alianza Editorial.

Vaquerizo-García, M. B.; Renedo-Mena, E. (2008). Herramientas para la Elaboración de Contenidos Didácticos en el Contexto e-Learning. Jenui.

Vaquerizo-García, M. B. (2011). Experiencia con Webquest y herramientas Web 2.0 en la evaluación de competencias transversales. Jenui.

\section{María Belén Vaquerizo-García}

Profesora Titular de Escuela Universitaria, Doctora, de la Universidad de Burgos, España. Ha recibido y participado en numerosos cursos de Formación Docente en el marco del EEES en la Universidad de Burgos, (obteniendo un CertificadoEspecialización en Formación-Docente-Marco-EEES). En este ámbito docente del EEES (metodologías, didácticas, tecnologías, formas de evaluación, tutorías,...) ha 